\title{
Long Term Survival in a Patient with Anaplastic Thyroid Carcinoma Treated with Cricotracheal Resection
}

\author{
Gregory Sayer $^{1}$, Douglas Sidell ${ }^{1}$, Joel A. Sercarz ${ }^{1,2^{*}}$ \\ ${ }^{1}$ Division of Head and Neck Surgery, University of California, Los Angeles, USA \\ ${ }^{2}$ Department of Surgery, Olive View-UCLA Medical Center, Los Angeles, USA \\ Email: *JSercarz@mednet.ucla.edu
}

Received May 16, 2012; revised June 30, 2012; accepted July 24, 2012

\begin{abstract}
Anaplastic thyroid cancer is an uncommon malignancy with a poor prognosis. Elderly patients are most commonly afflicted and survival past 3 years occurs in less than 5\% of patients. Management of these patients is challenging, and the importance of palliation, airway protection, and aggressive resection is debated. In this report, we describe a patient with anaplastic thyroid carcinoma who presented with respiratory distress due to invasion of the tracheal cartilage. The patient was managed with cricotracheal resection, total thyroidectomy and thyrotracheal anastomosis. The patient is currently disease free 3.5 years after resection and postoperative radiation therapy with interval neck dissection.
\end{abstract}

Keywords: Anaplastic; Thyroid Cancer; Survival; Treatment; Surgery

\section{Introduction}

Anaplastic carcinoma of the thyroid has a dismal prognosis, with a mean survival of less than 6 months [1]. The 5 year survival rate is $4 \%$ in patients 65 years or older [2]. With such a poor prognosis, the best treatment approach has been debated in the literature.

The recommended aggressiveness of treatment advocated in the literature varies as some authors recommend surgery for localized tumors, followed by postoperative adjuvant radiation therapy [3]. On the other hand, there is a consensus that patients with unresectable tumors or those with disseminated ATC should primarily be treated for palliation [5].

In this case report, we describe a 65-year-old female who presented with airway obstruction due to anaplastic carcinoma of the thyroid that arose in papillary carcinoma. The patient was treated with surgical resection including the upper trachea and anterior cricoid, in continuity with total thyroidectomy. This approach was selected because the tumor could be completely resectable with negative margins by cricotracheal resection. Postoperative radiation therapy has let to control of the disease. The case illustrates that with aggressive treatment, long-term survival with an excellent quality of life can be achieved, even when the tumor presents with airway involvement.

\section{Case Report}

The patient is a 65-year-old female who presented with a

${ }^{*}$ Corresponding author. 7-year history of a persistent lower neck mass. She later developed a 2-month history of wheezing and dyspnea on exertion. During her third Emergency Room admission for respiratory distress, a CT scan of the neck and upper chest revealed a $1.2 \mathrm{~cm} \times 8 \mathrm{~mm}$ subglottic tracheal mass with possible right true vocal cord involvement and a $2.2 \mathrm{~cm}$ right thyroid lobe mass (Figure 1). There was also a $2.4 \mathrm{~cm}$ right lateral compartment lymph node.

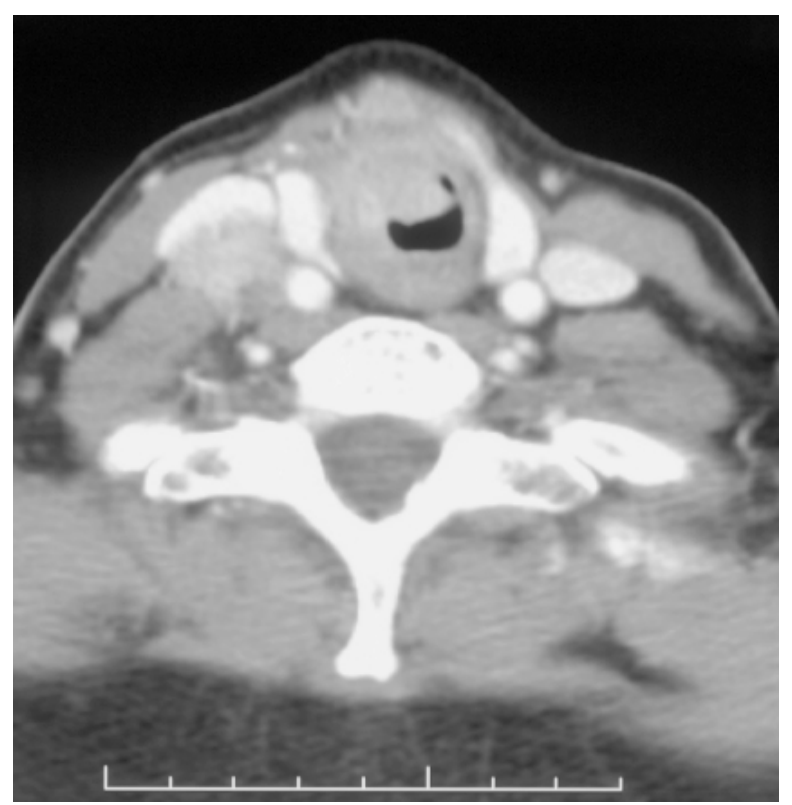

Figure 1. Axial CT scan of thyroid mass invading the airway. 
Rigid bronchoscopy was performed, indicating that the intraluminal component of the tracheal mass began $1 \mathrm{~cm}$ inferior to the true vocal cord and extended inferiorly within the lumen for a distance of $2.5 \mathrm{~cm}$. The tumor appeared to arise from the right side wall of the trachea. An $8 \mathrm{~mm} \times 8 \mathrm{~mm}$ section of pedunculated tumor was endoscopically removed. Microscopic analysis of the intraluminal specimen indicated the presence of anaplastic thyroid carcinoma arising out of well differentiated papillary thyroid carcinoma (Figure 2). Following bronchoscopic partial resection, her respiratory symptoms improved.

Shortly after diagnosis, the patient underwent resection
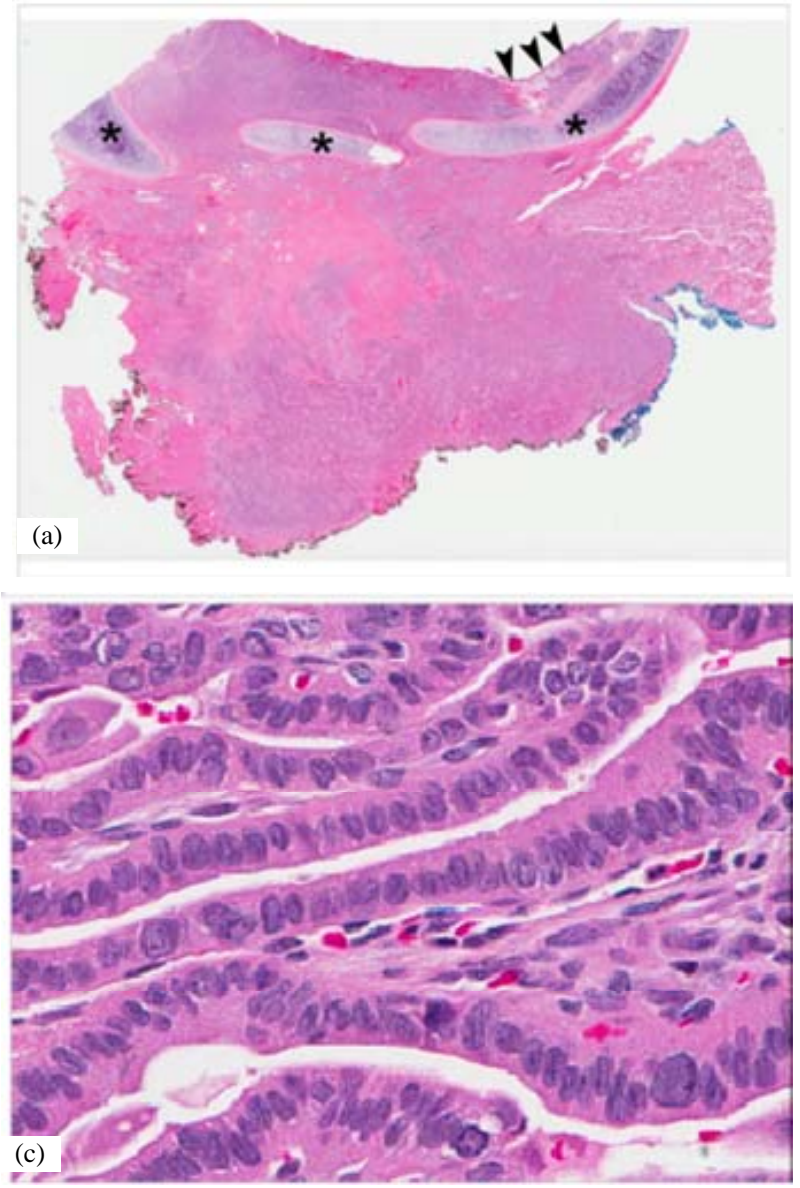

of the mass. The operation included a total thyroidectomy with right recurrent laryngeal nerve sacrifice and right paratracheal node dissection, resection of the upper trachea and cricoid, and anastomosis of the tracheal margin to the thyroid lamina. There were no complications and the patient was discharged on the seventh postoperative day with a tracheostomy.

Approximately 2.5 months after surgery, fiberoptic laryngoscopy indicated right vocal cord paralysis and a borderline airway with a narrow subglottis. The patient had no airway management difficulties and began successfully plugging the tracheostomy tube 10 months after surgery, and was decannulated 18 months after surgery.
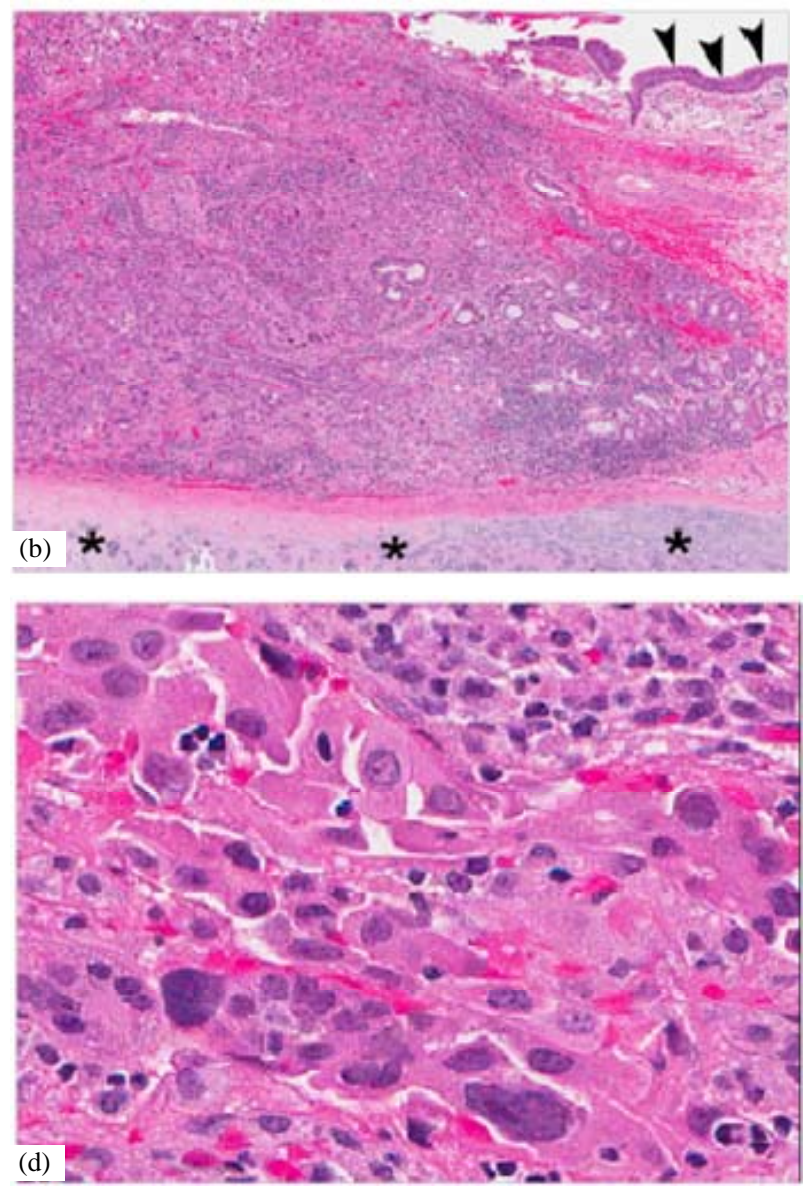

Figure 2. Undifferentiated (anaplastic) thyroid carcinoma arising from a well differentiated papillary thyroid carcinoma (hematoxylin-and-eosin stain). (a) Low power photomicrograph of tumor (original magnification, $3 \times$ ) extensively infiltrating trachea with erosion and ulceration of the tracheal mucosa. Residual tracheal mucosa (arrowheads) and plates of tracheal cartilage (asterisks) are noted; (b) Higher magnification of boxed area in previous figure (original magnification, 40 $\times$ ) showing an admixture of undifferentiated (anaplastic) thyroid carcinoma and well differentiated papillary thyroid carcinoma on the left side. The residual tracheal mucosa (arrowheads) is lined by ciliated pseudostratified columnar respiratory epithelium. Note the plate of tracheal cartilage at the bottom of the photomicrograph (asterisks); (c) High power photomicrograph (original magnification, $400 \times$ ) of well differentiated papillary thyroid carcinoma consisting of papillary structures with central fibrovascular cores lined by enlarged, elongated, and overlapping nuclei containing fine, powdery chromatin. Note the presence of multiple prominent intranuclear grooves; (d) High power photomicrograph (original magnification, 400 $\times$ ) showing area of undifferentiated (anaplastic) thyroid carcinoma consists of highly pleomorphic cells with abundant, eosinophilic, and dense cytoplasm, which imparts a squamoid appearance to the neoplastic cells. Note also the presence of prominent macronucleoli. 
Two years after the operation, a $1.5 \mathrm{~cm}$ enlarged level III right neck lymph node was found to have metastatic undifferentiated anaplastic carcinoma by fine needle aspiration. The patient underwent right neck dissection indicating a solitary $2 \mathrm{~cm} \times 1.5 \mathrm{~cm}$ mass consistent with metastatic anaplastic carcinoma. Twelve other nodes in the specimen were benign. An additional course of radiation therapy was delivered to the neck following surgery. The patient is currently alive and free of disease 3.5 years following her original surgery.

\section{Discussion}

Anaplastic thyroid cancer is usually a rapidly growing neoplasm with a poor prognosis. It generally occurs in the setting of previous thyroid pathology, such as goiter or papillary carcinoma, as in the present patient. The exact etiology is poorly understood and there has yet to be strong evidence identifying specific environmental or genetic factors, with the exception of iodine deficiency [6].

Anaplastic thyroid carcinoma can cause symptoms related to its involvement of adjacent structure such as the esophagus and trachea. Therefore, it should be considered a possible diagnosis in the setting of a thyriod mass accompanied by symptoms of airway obstruction, hoarseness and dysphagia. Diagnosis is obtained with either needle or incisional biopsy [7].

The role of surgical therapy for attempted cure is controversial, given the poor prognosis. Surgical intervention is indicated in anaplastic carcinoma when a complete resection is possible [7]. Factors that make a patient unresectable include prevertebral fascia invasion, carotid artery or other great vessel involvement, and extensive thoracic involvement. Postoperative adjuvant chemotherapy and radiation therapy are generally recommended. While certain positive prognostic factors have been agreed upon, such as tumor size less than $6 \mathrm{~cm}$ and female gender, studies on treatment outcomes are conflicting [5].

The anatomical location of anaplastic thyroid cancer and its rapid rate of growth raise the crucial issue of airway management in these patients [8]. In fact, airway distress can be one of the first presenting symptoms and is one of the most common causes of death from ATC as the endolarynx and trachea are affected $[9,10]$. Some experts have recommended palliation when the tumor involves the trachea or larynx as the prognosis is poor to begin with and significant post-operative morbidity often results [11]. In this case, we chose radical resection, including partial resection of the trachea and cricoid, because the tumor could be completely removed with this approach.

A study by Pierie et al. [12] indicated that complete resection for anaplastic carcinoma had statistically sig- nificant superior survival rates compared to incomplete debulking procedures. Incomplete debulking procedures also had statistically significant improvements in survival compared to those that did not receive surgery. More complete resection was found to prolong survival, but had a similar local recurrence rate as those treated with near-complete resection [13].

Although complete surgical resection may enhance local control of the tumor or even survival, the consequential morbidity may prohibit its use. Kihara et al. [14] concluded that for patients with advanced disease, requiring extended resections such as laryngectomy, palliation may be the best option. Chang et al. [11] also concluded that laryngectomy or tracheal resection is unjustified given the increase in morbidity. These studies raise the concern that extensive resection may negatively impact patients' quality of life and not achieve cure.

The use of radiation therapy in the treatment of anaplastic thyroid carcinoma also has been debated [15]. Chen et al. [16] showed that surgery alone for local disease without distant metastasis significantly improves survival while the benefit of additional radiation is less clear. Kebew et al. [3] support the use of radiation as an adjunct to surgery as it was a statistically significant independent variable associated with increased survival compared to surgery without radiation. Other studies, however, found that radiotherapy alone was an ineffective treatment modality [15]. Haigh et al. [17] found that radiotherapy with chemotherapy was also ineffective with a median survival of 3.3 months, which was not superior to a 3 month survival following palliative resection.

Combining treatment types is another potential option for patients with anaplastic carcinoma. Some studies support an aggressive, multi-modality treatment protocol to increase survival rates. Haigh et al. [17] found a statistically significant improvement in long-term survival in patients with complete resection or almost complete resection, with minimal residual disease, that then underwent chemotherapy and radiation compared to those undergoing palliative resection or chemotherapy and radiation without surgery. McIver et al. [13], on the other hand, found that a combination treatment plan with surgical resection, radiation, and chemotherapy did not demonstrate a statistically significant improvement in median survival of the 13 patients treated with this combination of therapy. However, $23 \%$ of those patients survived more than 1 year following treatment, which may suggest that some patients may be more benefit from aggressive treatment.

If combination treatment is associated with improved survival, the impact on the quality of life must also be considered. Patients treated with surgical resection, radiation, and chemotherapy had a statistically significant increase in grade 3 toxicity. Half of the patients treated 
with this multi-modality protocol required tube feeding secondary to radiation mucositis and esophagitis [18].

Airway management is another significant issue involved in treatment of these patients. Of all malignancies, anaplastic thyroid carcinoma most commonly requires tracheostomy as part of management [19]. Fewer prophylactic tracheostomies are being performed as there is an undue increase in morbidity and complications [16]. Tracheostomy is indicated only for patients with actual or impending airway obstruction. Securing the airway with a tracheostomy may be difficult when there is tumor bulk between the incision site on the skin and the anterior border of the trachea itself. With especially large tumors, a cricothyrotomy may be a more effective option to secure the airway [9]. Due to the anatomical location of our patient's tumor, a cricotracheal resection was performed; this operation, designed for use in subglottic stenosis, was anatomically suitable for the sites of tumor involvement.

Our patient had a common presentation of anaplastic thyroid carcinoma-respiratory distress secondary to significant airway involvement. Her uncomplicated clinical course and long term survival, however, are rare. An extensive resection and lymph node dissection, including cricotracheal resection, was well tolerated by the patient despite right vocal cord paralysis. Two years after this surgery, a lymph node demonstrated metastasis and was treated with lymph node dissection and radiation with no additionally complications. Our patient is a long term survivor has no evidence of disease 3.5 years since surgery. She has had no airway complications and is without tracheostomy or tube feeding.

\section{Conclusions}

Part of the challenge of designing treatment for such patients is the inherent bias in many of clinical studies of anaplastic carcinoma in the literature. Selection bias plays a role in the extent of treatment that patients receive. Patients with severe disease may not be able to tolerate a multi-modality treatment plan [20]. Also, complete surgical resection may be impossible for patients with very large or invading tumors compared to those with small ones. There is also a question of the impact of incidental versus evident anaplastic thyroid carcinoma as patients with incidental cancer have longer survival rates-likely because they have not become invasive to cause symptoms [20,21]. Most studies include incidental cancers in their statistical analyses. Additionally, many of these studies are retrospective and have small sample sizes. A large randomized control trial study could help elucidate the ideal treatment protocol and airway management of patients with anaplastic thyroid cancer.

While our patient responded well to aggressive surgical management, it was a somewhat unique anaplastic carcinoma that arose in a well differentiated papillary carcinoma. A larger series would be necessary to evaluate the role of cricotracheal resection for anaplastic carcinoma in general.

\section{REFERENCES}

[1] R. L. Neff, W. B. Farrar, R. T. Kloos and K. D. Burman, "Anaplastic Thyroid Cancer," Endocrinology and Metabolism Clinics of North America, Vol. 37, No. 2, 2008, pp. 525-538. doi:10.1016/j.ecl.2008.02.003

[2] C. Kosary, L. A. G. Ries, J. L. Young, G. E. Keel, et al., "SEER Survival Monograph: Cancer Survival Among Adults: U.S. Seer Program, 1988-2001, Patient and Tumor Characteristics,” Bethesda, National Cancer Institute, 2007.

[3] E. Kebebew, F. S. Greenspan, O. H. Clark, K. A. Woeber and A. McMillan, "Anaplastic Thyroid Carcinoma. Treatment Outcome and Prognostic Factors," Cancer, Vol. 103, No. 7, 2005, pp. 1330-1335. doi:10.1002/cncr.20936

[4] E. Brignardello, M. Gallo, I. Baldi, et al., “Anaplastic Thyroid Carcinoma: Clinical Outcome of 30 Consecutive Patients Referred to a Single Institution in the Past 5 Years,” European Journal of Endocrinology, Vol. 156, No. 4, 2007, pp. 425-430. doi:10.1530/EJE-06-0677

[5] R. K. Tan, R. K. Finley III, D. Driscoll, et al., "Anaplastic Carcinoma of the Thyroid: A 24-Year Experience,” Head \& Neck, Vol. 17, No. 1, 1995, pp. 41-48. doi:10.1002/hed.2880170109

[6] S. W. Wiseman, T. R. Loree, N. R. Rigual, et al., “Anaplastic Transformation of Thyroid Cancer: Review of Clinical, Pathologic, and Molecular Evidence Provides New Insights into Disease Biology and Future Therapy,” Head \& Neck, Vol. 25, No. 8, 2003, pp. 662-670. doi:10.1002/hed.10277

[7] W. R. Cornett, A. K. Sharma, T. A. Day, et al., “Anaplastic Thyroid Carcinoma: An Overview,” Current Oncology Reports, Vol. 9, No. 2, 2007, pp. 152-158. doi:10.1007/s11912-007-0014-3

[8] O. Nilsson, J. Lindeberg, J. Zedenius, et al. "Anaplastic Giant Cell Carcinoma of the Thyroid Gland: Treatment and Survival over a 25-Year Period,” World Journal of Surgery, Vol. 22, No. 7, 1998, pp. 725-730. doi:10.1007/s002689900460

[9] A. R. Shaha, "Airway Management in Anaplastic Thyroid Carcinoma,” The Laryngoscope, Vol. 118, 2008, pp. 11951198. doi:10.1097/MLG.0b013e3181726d36

[10] Y. Kitamura, K. Shimizu and M. Nagahama, et al., "Immediate Causes of Death in Thyroid Carcinoma: Clinicopathological Analysis of 161 Fatal Cases," Journal of Clinical Endocrinology and Metabolism, Vol. 84, No. 11, 1999, pp. 4043-4049. doi:10.1210/jc.84.11.4043

[11] H. Chang, K. Nam, W. Y. Chung and C. S. Park, “Anaplastic Thyroid Carcinoma: A Therapeutic Dilemma,” Yonsei Medical Journal, Vol. 46, No. 6, 2005, pp. 759-764. doi:10.3349/ymj.2005.46.6.759

[12] J. Pierie, A. Muzikansky, R. Gaz, W. Faquin and M. Ott, "The Effect of Surgery and Radiotherapy on Outcome of Anaplastic Thyroid Carcinoma,” Annals of Surgical On- 
cology, Vol. 9, No. 1, 2002, pp. 57-64. doi:10.1245/aso.2002.9.1.57

[13] B. McIver, I. D. Hay, D. F. Giuffrida, et al., "Anaplastic Thyroid Carcinoma: A 50-Year Experience at a Single Institution,” Surgery, Vol. 130, No. 6, 2001, pp. 1028-1034. doi:10.1067/msy.2001.118266

[14] M. Kihara, A. Miyauchi, A. Yamauchi and H. Yokomise, "Prognostic Factors of Anaplastic Thyroid Carcinoma," Surgery Today, Vol. 34, No. 5, 2004, pp. 394-398. doi:10.1007/s00595-003-2737-6

[15] C. Are and A. Shaha, "Anaplastic Thyroid Carcinoma: Biology, Pathogenesis, Prognostic Factors, and Treatment Approaches,” Annals of Surgical Oncology, Vol. 13, No. 4, 2006, pp. 453-464. doi:10.1245/ASO.2006.05.042

[16] J. Chen, J. D. Tward, D. C. Shrieve and Y. J. Hitchcock, "Surgery and Radiotherapy Improves Survival in Patients with Anaplastic Thyroid Carcinoma: Analysis of the Surveillance, Epidemiology, and End Results 1983-2002,” American Journal of Clinical Oncology, Vol. 31, No. 5, 2008, pp. 460-464. doi:10.1097/COC.0b013e31816a61f3

[17] P. I. Haigh, P. H. Ituarte, H. S. Wu, et al., "Completely Resected Anaplastic Thyroid Carcinoma Combined with Adjuvant Chemotherapy and Irradiation Is Associated with Prolonged Survival,” Cancer, Vol. 91, No. 12, 2001, pp. 2335-2342.

doi:10.1002/1097-0142(20010615)91:12<2335::AID-CN CR1266>3.0.CO;2-1

[18] A. T. Swaak-Kragten, J. H. de Wilt, P. I. Schmitz, M. Bontenbal and P. C. Levendag, "Multimodality Treatment for Anaplastic Thyroid Carcinoma-Treatment Outcome in 75 Patients,” Radiotherapy and Oncology, Vol. 92, No. 1, 2009, pp. 100-104. doi:10.1016/j.radonc.2009.02.016

[19] E. M. ElBashier, A. B. H. Widtalla and M. E. Ahmed, "Tracheostomy with Thyroidectomy: Indications, Management and Outcome: A Prospective Study," International Journal of Surgery, Vol. 6, No. 2, 2008, pp. 147-150. doi:10.1016/j.ijsu.2008.01.010

[20] N. Besic, M. Hocevar, J. Zgajnar, et al., "Prognostic Factors in Anaplastic Carcinoma of the Thyroid-A Multivariate Survival Analysis of 188 Patients," Langenbecks Archive of Surgery, Vol. 390, No. 3, 2005, pp. 203-208. doi:10.1007/s00423-004-0524-5

[21] K. Sugino, K. Ito, T. Mimura, et al., “The Important Role of Operations in the Management of Anaplastic Thyroid Carcinoma,” Surgery, Vol. 131, No. 3, 2002, pp. 245-248. doi:10.1067/msy.2002.119936 